\title{
Complex Permittivity Determination from Propagation Constant Measurements
}

\author{
Michael D. Janezic, Member, IEEE, and Jeffrey A. Jargon, Member, IEEE
}

\begin{abstract}
This letter presents a new transmission line method for measuring the complex permittivity of dielectric materials using propagation constant measurements. In contrast to previous methods, a network analyzer calibration is unnecessary since calibrated scattering parameters are not required. We use measurements in $X$-band waveguide to show that this technique compares well with the transmission/reflection and cylindrical cavity methods.
\end{abstract}

Index Terms-Dielectric constant, measurement, permittivity, propagation constant.

\section{INTRODUCTION}

$\mathbf{T}$ HE transmission/reflection (T/R) method is commonly used to measure the broad-band complex permittivity of dielectric materials [1], [2]. In this technique, a sample is inserted in a waveguide or coaxial transmission line. Although the sample must fill the entire cross section of the transmission line, the sample does not have to span the length of the transmission line. The complex permittivity of the sample is determined using iterative algorithms from calibrated scattering parameter measurements.

We propose a new method that uses measurements of the propagation constant rather than calibrated scattering parameters for determining the complex permittivity of a sample. In this method, two waveguide transmission lines of different lengths are filled completely with a dielectric material. The waveguides must have identical cross sections but different lengths. The complex propagation constant is determined from uncalibrated scattering parameter measurements of both waveguide transmission lines [3]. Since both waveguides are filled completely with the material, the complex permittivity of the sample is determined.

The following sections outline the theory of this new measurement method and present measurements that compare favorably with the T/R and cylindrical cavity methods.

\section{THEORY}

\section{A. Propagation Constant}

We use the multiline method [3], [4] for determining the propagation constant from uncalibrated scattering parameter measurements of two transmission lines. We define a measured cascade matrix of transmission line $i$ in terms of the measured

Manuscript received September 25, 1998.

The authors are with the National Institute of Standards and Technology, Boulder, CO 80303 USA.

Publisher Item Identifier S 1051-8207(99)02650-1. uncalibrated scattering parameters

$$
M^{i}=\frac{1}{S_{21_{i}}}\left[\begin{array}{cc}
\left(S_{12_{i}} S_{21_{i}}-S_{11_{i}} S_{22_{i}}\right) & S_{11_{i}} \\
-S_{22_{i}} & 1
\end{array}\right] .
$$

The measured cascade matrix can also be written as

$$
M^{i}=X T^{i} Y
$$

where cascade matrices $X$ and $Y$ represent imperfections of the network analyzer and the effects of the cables connected to the transmission line. We assume that $X$ and $Y$ are unchanged for each transmission line connection to the network analyzer. $T^{i}$ is the cascade matrix for an ideal transmission line $i$

$$
T^{i}=\left[\begin{array}{cc}
e^{-\gamma l_{i}} & 0 \\
0 & e^{\gamma l_{i}}
\end{array}\right]
$$

where $\gamma$ is the propagation constant and $l_{i}$ is the length of transmission line $i$. The measured cascade matrices of two transmission lines $i$ and $j$ of differing lengths can be combined into an eigenvalue equation

$$
M^{i j} X=X T^{i j}
$$

where

$$
M^{i j}=M^{j}\left[M^{i}\right]^{-1}
$$

and

$$
T^{i j}=T^{j}\left[T^{i}\right]^{-1} .
$$

Since $T^{i j}$ is diagonal, its diagonal elements are the eigenvalues of $T^{i j}$ and $M^{i j}$. The two eigenvalues $\lambda_{1 M}^{i j}$ and $\lambda_{2 M}^{i j}$ of $M^{i j}$ are

$$
\begin{aligned}
\lambda_{1 M}^{i j}, & \lambda_{2 M}^{i j} \\
& =\frac{\left(M_{11}^{i j}+M_{22}^{i j}\right) \pm \sqrt{\left(M_{11}^{i j}-M_{22}^{i j}\right)^{2}+4 M_{12}^{i j} M_{21}^{i j}}}{2}
\end{aligned}
$$

and the two eigenvalues $\lambda_{1 T}^{i j}$ and $\lambda_{2 T}^{i j}$ of $T^{i j}$ are

$$
\lambda_{1 T}^{i j}, \lambda_{2 T}^{i j}=e^{ \pm \gamma\left(l_{j}-l_{i}\right)} .
$$

We combine (7) and (8) to solve for the propagation constant

$$
\gamma=\frac{\ln \left(\lambda^{i j}\right)}{l_{i}-l_{j}}
$$

where $\lambda^{i j}$ is the average of the two eigenvalues

$$
\lambda^{i j}=\frac{1}{2}\left[\lambda_{1 M}^{i j}+\frac{1}{\lambda_{2 M}^{i j}}\right] .
$$




\section{B. Complex Permittivity}

We can divide the complex propagation constant into its real and imaginary parts

$$
\gamma(\omega)=\alpha(\omega)+j \beta(\omega)
$$

where $\alpha$ is the attenuation factor and $\beta$ is the phase factor. For a $\mathrm{TE}_{10}$ mode in rectangular waveguide, we can relate the real part of the sample permittivity $\epsilon_{s}^{\prime}$ to the phase constant $\beta$ [5] by

$$
\epsilon_{s}^{\prime}=\frac{\beta^{2}+\left(\frac{\pi}{a}\right)^{2}}{\omega^{2} \mu_{0} \epsilon_{0}}
$$

where

$$
\begin{array}{ll}
a & \text { longer width of the waveguide; } \\
\omega & \text { radian frequency; }
\end{array}
$$$$
\mu_{0}, \epsilon_{0} \quad \text { free space permeability and permittivity. }
$$

If we neglect the conductive losses in the metal waveguide, we can relate the imaginary part of the sample permittivity $\epsilon_{s}^{\prime \prime}$ to the attenuation constant $\alpha$ [5] by

$$
\epsilon_{s}^{\prime \prime}=\frac{2 \alpha \epsilon_{s}^{\prime}}{k} \sqrt{1-\left(\frac{\lambda}{2 a}\right)^{2}}
$$

where

$$
\lambda=\frac{2 \pi}{k}
$$

and

$$
k=\omega \sqrt{\mu_{0} \epsilon_{0} \epsilon_{s}^{\prime}} .
$$

So, from uncalibrated scattering parameter measurements, we determine the complex permittivity of a material within two waveguide transmission lines.

\section{Measurements AND COMPARISION}

A cross-linked polystyrene material was machined to fill two $X$-band waveguide transmission lines of lengths 25.4 and $10.0 \mathrm{~mm}$. We measured the complex propagation constant from 8 to $12 \mathrm{GHz}$ and calculated the complex permittivity of the material using (12) and (13). For comparison, we measured the complex permittivity of the 25.4-mm sample using the T/R technique [1]. From the same batch of crosslinked polystyrene, we machined a sample for the NIST 60-mm cylindrical cavity resonator [6]. This fixture provides a more accurate measurement of the sample permittivity at a single frequency of $10 \mathrm{GHz}$. Figs. 1 and 2 present the real and imaginary parts of the sample permittivity for all three methods. For $\epsilon_{s}^{\prime}$, the propagation constant method falls within the uncertainty bounds of the other two techniques. For $\epsilon_{s}^{\prime \prime}$, the propagation constant method is within the uncertainty bound of the T/R method, but not of the cylindrical resonator. This is not unexpected since resonator techniques are more accurate than transmission line methods for characterizing the dielectric losses of materials.

One uncertainty in the propagation constant measurement is the error due to unwanted air gaps between the material and the transmission line walls. In waveguide transmission lines, this

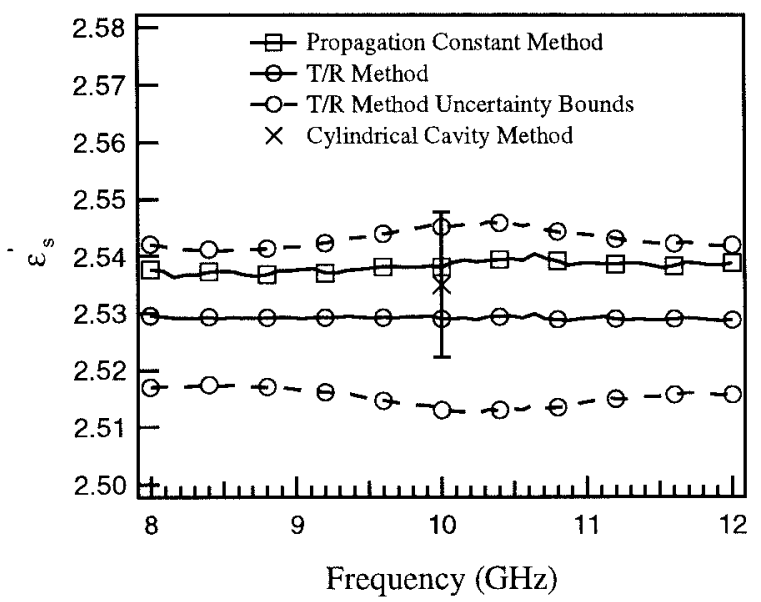

Fig. 1. Real part of cross-linked polystyrene permittivity $\epsilon_{s}^{\prime}$ as determined by the propagation constant, T/R, and cylindrical cavity methods.

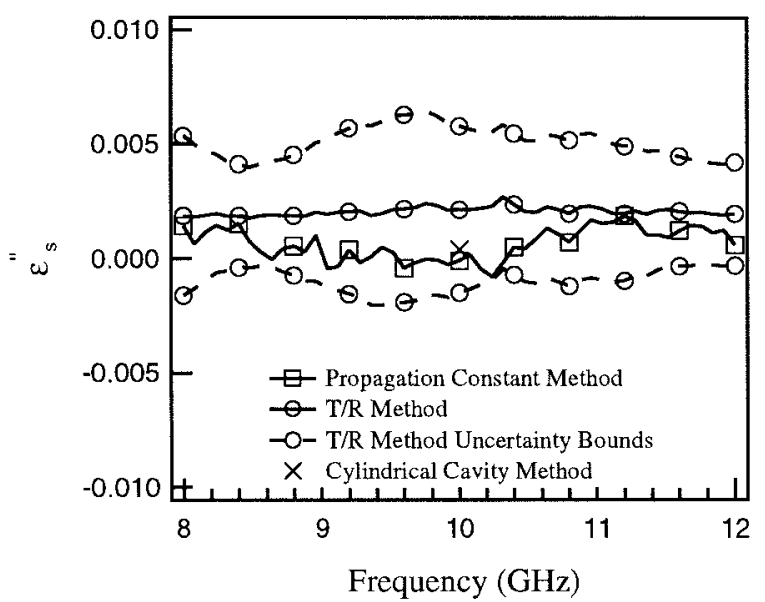

Fig. 2. Imaginary part of cross-linked polystyrene permittivity $\epsilon_{s}^{\prime \prime}$ as determined by the propagation constant, T/R, and cylindrical cavity methods. The combined standard uncertainty in the cylindrical cavity measurement is \pm 0.0001 and does not appear on this scale.

error is small since the electric field at two of the conductive walls is nearly zero. However, for coaxial transmission lines, the air gap between the toroidal sample and the inner conductor cannot be neglected since the electric field intensity is large near the inner conductor [2].

\section{CONCLUSIONS}

We have shown that the propagation constant method can accurately determine the complex permittivity of dielectric materials. This method compares favorably with the $T / R$ technique, with the advantages of not needing network analyzer calibration and a simple expression for the complex permittivity. A drawback of the propagation constant method is that it requires a second sample of differing length.

\section{ACKNOWLEDGMENT}

The authors would like to thank D. DeGroot, J. Baker-Jarvis, and R. Marks for their helpful discussions. 


\section{REFERENCES}

[1] J. Baker-Jarvis, E. Vanzura, and W. Kissick, "Improved technique for determining complex permittivity with the transmission/reflection method," IEEE Trans. Microwave Theory Tech., vol. 38, pp. 1096-1103, Aug. 1990.

[2] J. Baker-Jarvis, M. Janezic, J. Grosvenor, and R. Geyer, "Transmission/reflection and short-circuit line methods for measuring permittivity and permeability,” Natl. Inst. Stand. Technol., Tech. Note 1355-R, 1993.
[3] R. Marks, "A multiline method of network analyzer calibration," IEEE Trans. Microwave Theory Tech., vol. 39, pp. 1205-1215, July 1991.

[4] D. DeGroot, D. Walker, and R. Marks, "Impedance mismatch effects on propagation constant measurements," in IEEE 5th Topical Meeting on Electrical Performance of Electronic Packaging, Napa, CA, Oct. 1996.

[5] S. Ramo, J. Whinnery, and T. Van Duzer, Fields and Waves in Communications Electronics. New York: Wiley, 1984.

[6] E. Vanzura, R. Geyer, and M. Janezic, "The NIST 60-millimeter diameter cylindrical cavity resonator: Performance for permittivity measurements," Natl. Inst. Stand., Technol. Tech. Note 1354, 1993. 